\title{
An Efficient Transmission Scheme for Media Content Distribution Platform
}

\author{
Feifei Wang \\ Digital Engineering Center \\ Communication University of China \\ Beijing, China \\ wangfeifei@cuc.edu.cn \\ Yichao Yin \\ Grus (Beijing) Technology Co., LTD. \\ Beijing, China \\ yinyichao@grustv.com
}

\author{
Hongfei Guo, Jie Kang \\ Communication Technology Bureau \\ XinHua News Agency \\ Beijing, China \\ guohongfei@xinhua.org
}

\begin{abstract}
The selection of transmission schemes of content distribution platforms is usually decided by three key factors: application type, channel quality and network topology. To simplify the design of such platforms, this paper proposes an efficient transmission scheme which is adapted to most scenarios. Two key components are introduced to make the scheme more adaptable and efficient. Simulation and application results in Xinhua News Agency show that this scheme has a much higher efficiency than the traditional ones
\end{abstract}

Keywords - content distribution, TCP, data carousel, fountain code, Reliable UDP.

\section{INTRODUCTION}

With the rapid development of multimedia technology, it makes out several new features for multimedia content. More video resources with higher resolution results in a higher data transmission demand and meanwhile, the size of a single file becomes much larger. The main goal of the content distribution platforms is to deliver the resources as fast as possible. In order to obtain the best efficiency, many transmission technology are proposed according to the application type, channel quality and network topology, such as FTP, P2P, CDN in the bidirectional link and the data carousel protocol, packet-level FEC in unidirectional link. However, the traditional transmission schemes are easily affected by network packet loss rate and latency and have low efficiency. Although the average bandwidth is increased by several times during the past five years, most of it is wasted. A lot of research have been done to improve the efficiency of the basic protocols [1] [2] [3] [4], but they are still not efficient enough to satisfy the transmission requirement of a rapidly growing volume of data.

In this paper, we first investigate the application scenarios of the data exchange businesses in Xinhua News Agency and summarize five typical models of a common content distribution platform. Then we put forward an efficient transmission scheme which contains two key components and also give a suggestion for the selection. Finally, test results are obtained and compared with some traditional schemes.

\section{SUMMARY OF APPLICATION SCENARIOS}

Content distribution has a very close relationship with human daily life for both enterprises and individuals. Here are some applications examples: on-demand or live video services, file downloading or uploading, advertisement pushing, news manuscript delivery, etc. The functions and structures may vary from platform to platform according to the application scenarios. However, we only need to consider transmission related differences to simplify the problem. Therefore an investigation in XinHua has been conducted and five typical distribution models are summarized.

The main application scenarios of content distribution in XinHua are listed as follows:

- S1: News products distribution via private network or internet with good network quality.

- S2: News products distribution via internet with bad network quality.

- S3: News products distribution via satellite.

- S4: Uploads of news material collected by reporters.

- S5: Data exchange between different processing platforms.

- S6: Program update of the outdoor video screens.

- S7: Acceleration of transmission for cloud-based services.

- S8: Emergency manuscripts transmission by HF communication.

By comparing the application scenarios, the transmission related differences can be concluded as three features: transmission type, network type, and network quality. The transmission type is upload (U) and download (D). The network type decides the link is unidirectional (UNI) or bidirectional (BI), and the number of the senders and receivers, which is represented as point-to-point (P2P), point-to- 
multipoint (P2M), multipoint-to-point (M2P) and multipointto-multipoint (M2M). The network quality contains three parameters: bandwidth, packet loss rate and latency.

The detail is shown in Table I. As the internet environment is very complex, we suppose two scenarios: S1 has a short transmission distance with good network quality in national wide, while S2 has a long transmission distance with bad network quality in worldwide.

TABLE I. TRANSMisSion SCENARIo FEATURES

\begin{tabular}{|c|c|c|c|c|c|}
\hline \multirow{3}{*}{$\begin{array}{l}\text { Applic } \\
\text { ation } \\
\text { Scenar } \\
\text { ios }\end{array}$} & \multicolumn{5}{|c|}{ Transmission Scenario Features } \\
\hline & \multirow{2}{*}{$\begin{array}{c}\text { Transmi } \\
\text { ssion } \\
\text { Type } \\
\end{array}$} & \multirow{2}{*}{$\begin{array}{l}\text { Network } \\
\text { type }\end{array}$} & \multicolumn{3}{|c|}{ Network Quality } \\
\hline & & & $\begin{array}{l}\text { Bandwid } \\
\text { th(Mbps) }\end{array}$ & $\begin{array}{c}\text { Packet } \\
\text { Loss Rate }\end{array}$ & $\begin{array}{c}\text { Latency } \\
(\mathrm{ms})\end{array}$ \\
\hline S1 & $\mathrm{U}$ & BI, P2P & $>10$ & $<1 \%$ & $<100$ \\
\hline S2 & $\mathrm{U}$ & BI, P2P & $<10$ & $>0.5 \%$ & $>200$ \\
\hline $\mathrm{S} 3$ & $\mathrm{U}$ & UNI, P2M & $8 \sim 15$ & $<0.01 \%$ & 270 \\
\hline S4 & $\mathrm{D}$ & BI, P2P & $<10$ & $<2 \%$ & $<100$ \\
\hline S5 & $\mathrm{U} \& \mathrm{D}$ & $\mathrm{BI}, \mathrm{P} 2 \mathrm{P}$ & $>15$ & $<0.01 \%$ & $<10$ \\
\hline S6 & $\mathrm{U}$ & BI, P2P & $<10$ & $<1 \%$ & $<100$ \\
\hline S7 & $\mathrm{U} \& \mathrm{D}$ & BI, P2P & $>8$ & $<1 \%$ & $<100$ \\
\hline S8 & $\mathrm{U}$ & UNI, P2M & $<0.03$ & $>30 \%$ & $>10$ \\
\hline
\end{tabular}

Based on the features of the transmission scenarios, some transmission scenarios can be grouped. We have three basic rules to do the grouping:

- Whether has a feedback channel.

- Network quality is more or less the same.

- Number of the receiving terminals.

In practical use, network quality can be divided into good ( $\mathrm{G}$, low packet loss rate, low latency and stable), fair ( $\mathrm{F}$, most cases), bad ( $\mathrm{B}$, high packet loss rate, high latency and not stable). The large number (usually more than 500) of receiving terminals may cases the ACK implosion problem so that we make it a separate one. Thus five typical distribution models are summarized as shown in Table II.

TABLE II. Typical Models of Distribution Platforms

\begin{tabular}{|c|c|c|c|c|}
\hline \multirow{2}{*}{ Models } & \multicolumn{4}{|c|}{ Features } \\
\cline { 2 - 5 } & $\begin{array}{c}\text { Feedback } \\
\text { Channel }\end{array}$ & $\begin{array}{c}\text { Network } \\
\text { Quality }\end{array}$ & $\begin{array}{c}\text { Number of } \\
\text { Terminals }\end{array}$ & $\begin{array}{c}\text { Application } \\
\text { Scenario }\end{array}$ \\
\hline M1 & N & - & - & S3, S8 \\
\hline M2 & Y & VB & Small & S2 \\
\hline M3 & Y & F & Small & S1, S4, S7 \\
\hline M4 & Y & VG & Small & S5 \\
\hline M5 & Y & - & Large & S6 \\
\hline
\end{tabular}

Most of other transmission scenarios can be classified into one of these models.

\section{DESIGN OF ThE TRANSMISSION SCHEME}

The main transmission protocols used by current schemes are TCP and data carousel protocol (most based on UDP). For bidirectional connections, TCP protocol is used to ensure that each packet of data is correctly received. For unidirectional connections, redundant data must be transferred to resist the packet loss. It may transfer the same data more than two times with the data carousel protocol.

Disadvantages of transmission schemes based on these protocols are discussed first in this section. And then a more adaptable and efficient scheme with two key components is described in detail.

\section{A. Disadvantage of Existing transmission schemes}

FTP and HTTP are most common TCP-based methods of content distribution platforms. The data are stored on a center server and the users apply for a download separately. As having been widely discussed, they are easily affected by network latency. It takes maximum 3 seconds to renegotiate for the sequence to restart from the missing packet and discarding all the subsequent data, which means the transmission seems like "disconnected" and nothing is transferred within the period. As a result, it greatly decreases the throughput of the network in packet-loss environment [5].

The standard format for the data carousel is defined in the Digital Storage Media Command and Control (DSM-CC) toolkit in ISO/IEC 13818-6 [6], and commonly used in a unidirectional broadcast environment based on UDP. Without a feedback channel, the receiver is unable to request the retransmission of any missing data. The sender repeatedly delivers the same data in a continuous cycle to make up the missing packet at the cost of a large amount of duplicate packets, sometimes more than 99\%. This method is very sensitive to the packet loss rate and the file size. Even with a packet loss rate of $0.01 \%$ in the satellite network, a 100MB file can hardly be received correctly for the first time.

Most of the existing transmission schemes cannot deal with the latency and packet loss well due to the limitations of TCP or data carousel protocol. Thus the bandwidth is more or less wasted.

\section{B. Proposed Scheme}

To reduce the network capacity inefficiency, we proposed an adaptable and efficient scheme that can support all the models as shown in Table II.

Dynamically adjusting the congestion window may be a good method to improve the performance of TCP [7], but it's difficult to realize and implement. UDP offers some advantages over TCP, such as higher efficiency and more accurate transmission time, regardless of the reliability. By contrast, UDP is more potential to bring the bandwidth utilization up to its limit.

So our scheme uses UDP as a basic protocol, and introduces some advanced technic to improve or make sure the reliability. Both digital-fountain-code-based UDP (FUDP) and Reliable UDP (RUDP) are employed as two key components to achieve the best performance for each model.

The position which FUDP and RUDP reside in the fivelayer networking model is shown in Fig. 2. 


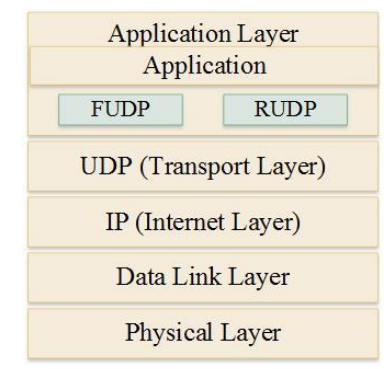

Fig. 1. Modified networking model

\section{1) Component FUDP}

The digital fountain codes were first proposed as LT codes [8] in 1998 by Michael Luby et al. With these codes, the receiver is able to recover the source block from any set of the encoded symbols, which has only slightly more packets in number than the source symbols with a high probability. Due to their "rateless" property and channel independent performance, fountain codes can achieve very high bandwidth utilization in the unidirectional connections. Raptor codes [9] are more advanced fountain codes with linear encoding and decoding complexity. For a source block with maximum 8192 packets, only 30 extra packets (no more than $0.4 \%$ ) are needed to recover the data with failure probability of $1 \mathrm{e}-8$.

The schematic diagram of Raptor codes is given in Fig. 2. The encoder generates $n$ redundant packets by using the $m$ source packets and then send all the $m+n$ encoding packets. Whenever the receiver collects enough packets (might be $m$ ), the decoder can recover the missing source packets. The decoder only cares how many packets it receives rather than which packet it gets.

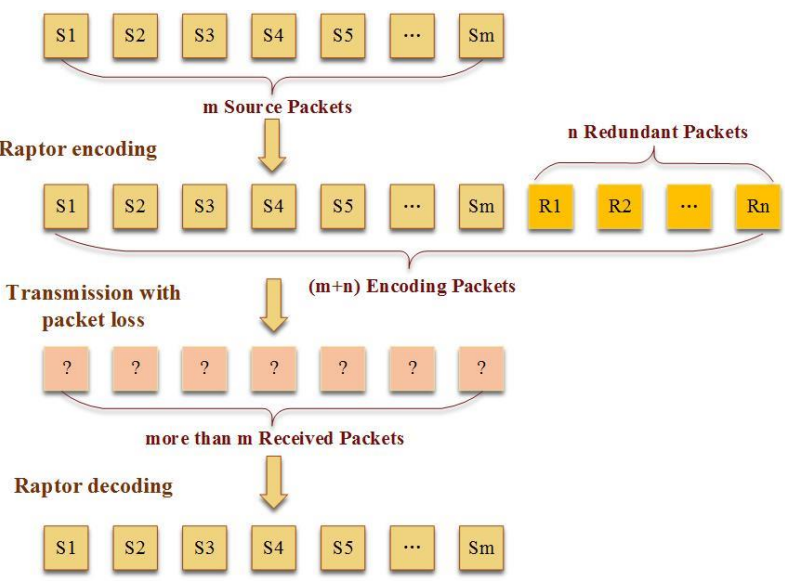

Fig. 2. Schematic diagram of Raptor codes.

In our scheme, component FUDP uses UDP as a transport protocol and encodes data with Raptor codes in application layer to generate enough encoding packets that can cope with the packet loss. We defines a header structure in Table III. It makes FUDP can support files with a maximum size of 700GB and variable parameters for different applications.
TABLE III. HEADER STRUCTURE OF FUDP

\begin{tabular}{|c|c|c|}
\hline Parameter & Meaning & Length \\
\hline TBN & $\begin{array}{c}\text { total number of source blocks } \\
\text { divided }\end{array}$ & 2 Bytes \\
\hline K & $\begin{array}{c}\text { the number of source packets in a } \\
\text { single block }\end{array}$ & 2 Bytes \\
\hline EBI, & encoding Block ID & 2 Bytes \\
\hline EPI & encoding packet ID & 2 Bytes \\
\hline
\end{tabular}

\section{2) Component RUDP}

Although the Raptor codes have preferable channel independent performance, their much higher computational complexity sets a minimum requirement of computers on both server and client sides. In that case, RUDP, which has the same level of complexity with TCP, is introduced as another key component.

RUDP is proposed in 1999 on the basis of RDP as an IETF Internet-Draft [10]. For the purpose of reliable transmission, RUDP uses a synchronization message (SYN) which contains port and host address to establish a connection. The process is much the same as a three-way handshake used in TCP. The SYN message also includes the negotiable parameters of the connection. RUDP indexes each packet and employs acknowledgements to monitor the missing data. As the protocol doesn't give the parameters that can achieve best performance in most situations, RUDP to a large extent is a task-specific protocol. Both Cisco and Microsoft have released RUDP versions of their own within their stacks for specific tasks.

In our scheme, RUDP only needs to take care of part of the application scenarios, so we use a header as shown in Fig. 3.

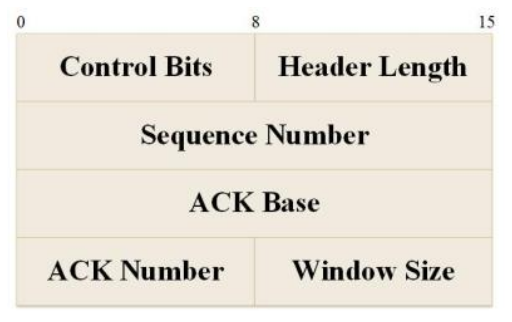

Fig. 3. RUDP header

Different from the original protocol, our scheme uses ACK base and ACK number to record the lost packets exactly. ACK base is the first lost packet and the ACK number indicates how many packets are not received. In this way, the retransmission doesn't start from the first disordered point, but the point the packets are really lost.

\section{Modified FUDP for Bidirection Connections}

Although FUDP can obtain a relatively high success rate, it can't guarantee 100 percent success rate by using a finite of redundant packets. As the receiver doesn't care which packets to get, it only needs to acknowledge the information whether it has collected enough packets. Meanwhile, for a dynamically changing bandwidth, congestion control is also necessary.

In our scheme, a TCP connection is used to transfer the acknowledgements, whicn contains the EBI and the number of 
received packets belonging to the block. The ACKs are transferred at least once for each data block. A retransmission starts when ACKs arrived. To realize the congestion control, the sender dymactically adjusts the number of redundant packets to generate and deliver according to the ACKs.

Based on our test results, averagely only 1 or 2 ACKs are transferred every 10 seconds with a bandwidth of $10 \mathrm{Mbps}$. So the loss of the efficiency is very limited.

\section{Selection of The Components}

As the network environment of the contents distribution platforms is much too complicated, it's difficult to find a universal method for all the transmissions. In this paper, FUDP and RUDP are assigned to different application scenarios to make full use of their respective advantages.

To help make the decision, Table IV give a comparison of the two components. RUDP has a slightly lower efficiency but lower computational complexity. So it's a better choice for a transmission with fair or good network quality. As the RUDP has more ACKs, it has a better performance in congestion control in the bidirectional networks. Compared with RUDP, FUDP can support the unidirectional network and broadcast transmission much better and is very suitable for the satellite transmissions.

TABLE IV. COMPARISON OF THE COMPONENTS

\begin{tabular}{|c|c|c|}
\hline Parameter & FUDP & RUDP \\
\hline Efficiency & Slightly high & Slightly low \\
\hline $\begin{array}{c}\text { Computational } \\
\text { complexity }\end{array}$ & High & Low \\
\hline Overhead & Slightly high & Slightly low \\
\hline Broadcast-support & Well support & support \\
\hline Congestion control & Fair & Good \\
\hline $\begin{array}{c}\text { Unidirectional } \\
\text { network-support }\end{array}$ & Well Support & Not support \\
\hline
\end{tabular}

In summary, in Table $\mathrm{V}$, we give a suggestion for the selection of the components by taking the five typical distribution models discussed in Section II for example.

TABLE V. Suggested Selection of Typical Models

\begin{tabular}{|c|c|c|}
\hline Model & Component & Reason \\
\hline M1 & FUDP & $\begin{array}{c}\text { Unidirectional } \\
\text { network }\end{array}$ \\
\hline M2 & FUDP & $\begin{array}{c}\text { Fewer } \\
\text { acknowledgements }\end{array}$ \\
\hline M3 & RUDP & $\begin{array}{c}\text { Low computational } \\
\text { complexity }\end{array}$ \\
\hline M4 & RUDP & Lower overhead \\
\hline M5 & FUDP & $\begin{array}{c}\text { Avoid ACK } \\
\text { implosion problem }\end{array}$ \\
\hline
\end{tabular}

\section{PERformance TeSt Results}

Both simulation and actual test are implemented in this paper.

\section{A. Simulation Test}

Figure 4 shows the goodput of the one-way transmissions using FUDP and data carousel protocol. To give a theoretical reference bandwidth, we define the maximum goodput $G_{\max }$ as following:

$$
G_{\max }=\frac{1-R_{\text {loss }}}{B}
$$

Where $R_{\text {loss }}$ represents the packet loss rate and $B$ represents the transmission bandwidth.

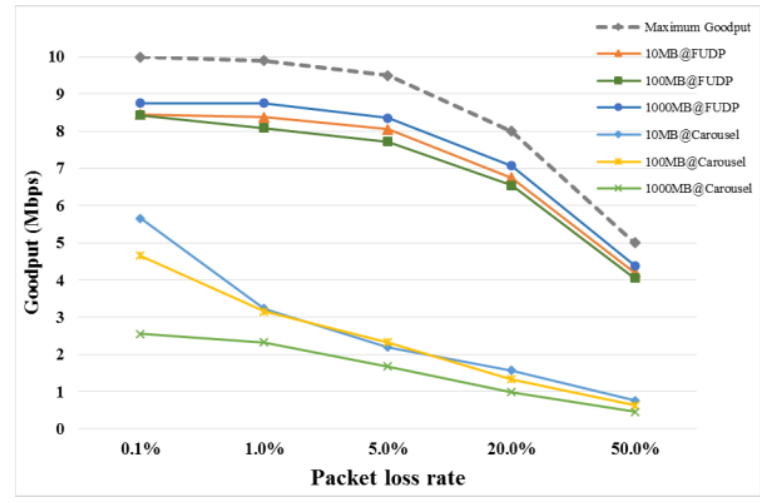

Fig. 4. Simulation results of FUDP

By employing FUDP, the transmission kept a very stable and high goodput when the pakcet loss rate increased from $0.1 \%$ to $50 \%$. Its average bandwidth utiliztation (nearly $90 \%$ ) is about 4 times higher than the scheme's with data carousel protocol.

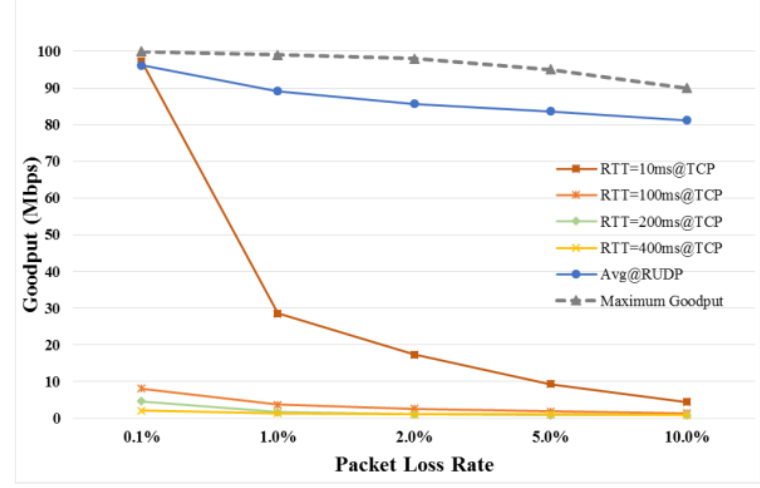

Fig. 5. Simulation results of RUDP

Based on two-way transmissions, the simlulation results of RUDP with respect to TCP is shown in Fig. 5. It can be seen that the performance of TCP is greatly affected by network latency and packet loss. The goodput quickly dropped from nearly $100 \mathrm{Mbps}$ to $30 \mathrm{Mbps}$ when the packet loss rate increased from $0.1 \%$ to $1 \%$. Cause there is little correlation between the performance of RUDP and network latency, we only give an average goodput with different RTT. RUDP exhibited a considerably higher transmission efficiency than TCP when the network quality was not very good. 


\section{B. Actual Test}

In order to verify the performance of our scheme in the practical application, a demo system of HD quality video distribution is realized in XinHua. By this system, HD videos are distributed to the branch agencies via satellite and internet. The bandwidth of the satellite transmission is fixed to $8 \mathrm{Mbps}$. The bandwidth of the internet transmission is set by users according to the actual network quality. A 5GB HD video is delivered for every test.

Table VI shows the test results of satellite transmissions. The packet loss rate is about $0.01 \%$ based on experience. The data is usually transferred twice by using existed system. Its goodput is less than $4 \mathrm{Mbps}$. When given a redundancy rate more than $1 \%$, our system achieves a 100 percent success rate for this test. Higher redundancy rate can improve the success rate but also causes a waste of bandwidth, because the fountain codes decoder only need about 30 more packets for a single data block.

TABLE VI. Actual Test Results via SATellite

\begin{tabular}{|c|c|c|c|}
\hline $\begin{array}{c}\text { Redundan } \\
\text { cy Rate }\end{array}$ & $\begin{array}{c}\text { Success } \\
\text { Rate }\end{array}$ & $\begin{array}{c}\text { Average data } \\
\text { size for } \\
\text { decoder }\end{array}$ & $\begin{array}{c}\text { Goodput } \\
\text { (Mbps) }\end{array}$ \\
\hline $0.50 \%$ & $98 / 100$ & $5649 \mathrm{MB}$ & 7.45 \\
\hline $1.00 \%$ & $100 / 100$ & $5648 \mathrm{MB}$ & 7.41 \\
\hline $5.00 \%$ & $100 / 100$ & $5649 \mathrm{MB}$ & 7.14 \\
\hline
\end{tabular}

Table VII shows the test results of internet transmissions. We have five receivers located in different provinces. The test based on an existed FTP system is first done as a reference for comparison. Our system showed a better performance than FTP.

TABLE VII. Actual Test Results VIA Internet

\begin{tabular}{|c|c|c|c|}
\hline $\begin{array}{c}\text { Location } \\
\text { of Receiver }\end{array}$ & $\begin{array}{c}\text { Bandwidth } \\
\text { (Mbps) }\end{array}$ & $\begin{array}{c}\text { Goodput of } \\
\text { FTP (Mbps) }\end{array}$ & $\begin{array}{c}\text { Goodput of our } \\
\text { System (Mbps) }\end{array}$ \\
\hline Nanjing & 16.2 & 11.2 & 15.8 \\
\hline Ningxia & 20 & 10.2 & 18.6 \\
\hline Shenzhen & 50 & 2.4 & 46.4 \\
\hline Yancheng & Not limited & 14.0 & 70.0 \\
\hline Taizhou & Not limited & 8.0 & 40.0 \\
\hline
\end{tabular}

\section{CONCLUSION}

In this paper, we proposed an efficient transmission scheme that is adapted to most application scenarios. This scheme introduced FUDP and RUDP as two key components to satisfy the requirement of content distribution platforms in both unidirectional and bidirectional transmissions. The comparison of the components was given to help users to make a quick selection. The simulation and actual test results indicated this scheme has much higher efficiency than the traditional schemes.

\section{ACKNOWLEDGMENT}

This work is supported by a grant from National Key Technology R\&D Program of China under contract No. 2014BAK10B01.

\section{REFERENCES}

[1] L. Seoung-Bum, A. Gahng-Seop and A. T. Campbell, "Improving UDP and TCP performance in mobile ad hoc networks with INSIGNIA," Communications Magazine, IEEE, vol. 39, pp. 156-165, 2001.

[2] L. Wanjiun, K. Chang-Jung and C. H. Chien, "Improving TCP performance in mobile networks," Communications, IEEE Transactions on, vol. 53, pp. 569-571, 2005.

[3] L. Feng and S. Jun, "An Interactive Service Platform Solution Based On Enhanced Data Carousel Scheme," Consumer Electronics, IEEE Transactions on, vol. 53, pp. 675-682, 2007.

[4] R. Fengyuan and L. Chuang, "Modeling and Improving TCP Performance over Cellular Link with Variable Bandwidth," Mobile Computing, IEEE Transactions on, vol. 10, pp. 10571070, 2011.

[5] S. Henna, "A Throughput Analysis of TCP Variants in Mobile Wireless Networks," in Next Generation Mobile Applications, Services and Technologies, 2009. NGMAST '09. Third International Conference on, Cardiff, Wales, 2009, pp. 279-284.

[6] ISO/IEC 13818-6, "Generic coding of moving pictures and associated audio information - part 6: Extension for digital storage media command and control (DSM-CC)," 1997.

[7] T. Kelly, "Scalable TCP: Improving performance in high speed wide area networks," ACM SIGCOMM Computer Communication Review, vol. 33, pp. 83-91, 2003.

[8] M. Luby, "LT codes," in Foundations of Computer Science, 2002. Proceedings. The 43rd Annual IEEE Symposium on, 2002, pp. 271-280.

[9] A. Shokrollahi, "Raptor codes," Information Theory, IEEE Transactions on, vol. 52, pp. 2551-2567, 2006.

[10] T. Bova and T. Krivoruchka, "Reliable UDP Protocol," IETF Internet-Draft, 1999. 